\title{
Role of the Wnt/ $\beta$-catenin signaling pathway in the response of chondrocytes to mechanical loading
}

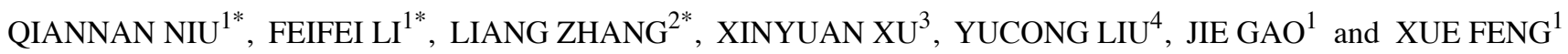 \\ ${ }^{1}$ State Key Laboratory of Military Stomatology, Department of Orthodontics, School of Stomatology, \\ The Fourth Military Medical University, Xi'an, Shaanxi 710032; ${ }^{2}$ Department of Stomatology, Hospital 323 of \\ The People's Liberation Army, Xi'an, Shaanxi 710045; ${ }^{3}$ Department of Biochemistry and Molecular Biology, \\ School of Stomatology, The Fourth Military Medical University, Xi'an, Shaanxi 710032; ${ }^{4}$ Department of Stomatology, \\ The First People's Hospital of Shuangliu County, Chengdu, Sichuan 610200, P.R. China
}

Received July 17, 2015; Accepted January 5, 2016

DOI: $10.3892 /$ ijmm.2016.2463

\begin{abstract}
In order to better understand the mechanisms by which chondrocytes respond to mechanical stimulation, ATDC5 mouse embryonic carcinoma cells were induced to differentiate into chondrocytes and then exposed to mechanical loading. To specifically elucidate the role of this pathway, the localization and expression of proteins involved in the $\mathrm{Wnt} / \beta$-catenin signaling pathway were observed. Chondrogenic-differentiated ATDC5 cells were exposed to a $12 \%$ cycle tension load for 1,2 , 4 , or $8 \mathrm{~h}$. At each time point, immunofluorescence staining, western blot analysis, and qPCR were used to track the localization of $\beta$-catenin and glycogen synthase kinase-3 $\beta$ (GSK-3 $\beta$ ) expression. In addition, the mRNA expression of Wnt3a, disheveled homolog 1 (Dvl-1), GSK-3 $\beta$, and collagen type II were also detected. Activation of the Wnt/ $\beta$-catenin signaling pathway was investigated in cells treated with Dickkopf-related protein 1 (DKK-1). $\beta$-catenin and GSK-3 $\beta$ protein expression increased initially and then decreased over the mechanical loading period, and the corresponding mRNA levels followed a similar trend. After application of the inhibitor DKK-1, Wnt/ $\beta$-catenin signaling was suppressed, and the mRNA expression of collagen II was also reduced. Thus, stimulation
\end{abstract}

Correspondence to: Dr Xue Feng, State Key Laboratory of Military Stomatology, Department of Orthodontics, School of Stomatology, The Fourth Military Medical University, 145 Changle West Road, Xi'an, Shaanxi 710032, P.R. China

E-mail: september_nqn@163.com

*Contributed equally

Abbreviations: DKK-1, Dickkopf-related protein 1; DMSO, dimethyl sulfoxide; GSK-3 $\beta$, glycogen synthase kinase-3 $\beta$; LRP5/6, lipoprotein receptor-related protein 5/6; TMJ, temporomandibular joint; MCC, mandibular condylar chondrocyte; MTT, 3-(4,5-dimethylthiazol-2-yl)2,5-diphenyltetrazolium bromide

Key words: mechanical strain, chondrocyte, $\beta$-catenin, p-glycogen synthase kinase-3 $\beta$ of chondrocytes with mechanical strain loading is associated with the translocation of active $\beta$-catenin from the cytoplasm to the nucleus.

\section{Introduction}

The temporomandibular joint (TMJ) is the only bilateral linkage joint in the human body, and it is characterized by stability and mobility. Activities such as mastication, swallowing, talking, and facial expressions are controlled by the TMJ and the associated muscles. The condylar process is an important developmental zone of the mandible, and the development of the TMJ is closely related to functional stimulation and plays an important role in oral-maxillary facial development. The growth and development of chondrocytes within the TMJ are closely related to functional mechanical stimulation. The TMJ disc is located between the mandible and the skull and plays a critical role in mandibular movements, functioning as a stress absorber (1). During various functional movements, such as chewing and talking, or some dysfunctional movements such as bruxism, the disc is continuously subjected to biomechanical loading in the form of tensile, compressive, and shear stress from different directions (2). Moreover, during orthodontic treatment, patients are often subjected to intermaxillary traction. The cartilage matrix as well as chondrocytes are responsible for responding to mechanical stress and maintaining the function of articular joints. During clinical treatment, intermaxillary traction is highly effective for achieving tooth movement, but may also cause a series of undesired results related to the force-induced response, such as temporomandibular disorders (3). One study reported that endochondral bone formation is reduced, with restriction of mandibular movements, during the chondylar cartilage development process (4). Mandibular condylar chondrocytes (MCCs) are the main type of chondylar chondrocytes, and they play an important role in maintaining cartilage homeostasis. MCC proliferation, differentiation, and apoptosis directly influence the mechanical characteristics and functional status of the joint. However, despite advances in our understanding of the molecular mechanisms of cartilage development and repair, the mechanical signals that regulate cartilage repair remain poorly understood (5-7). 
A previous study by Kronenberg (8) found that several signaling pathways are activated during the development and growth of chondrocytes, including the Sox 9, transforming growth factor (TGF) $-\beta$, and Wnt signaling pathways. Moreover, a study by Maruyama et al (9) on systemic skeletal diseases and metabolic diseases focused on the Wnt signaling pathway, and it has been confirmed that the Wnt signaling pathway is associated with biological force stimulation (10). The Wnt signaling pathway plays an important role in the regulation of chondrogenic differentiation and hypertrophy $(11,12)$. Three distinct intracellular Wnt signaling cascades are well known at present: the Wnt/ $\beta$-catenin pathway (the canonical Wnt signaling pathway), the c-Jun N-terminal kinase (JNK) pathway, and the $\mathrm{Wnt} / \mathrm{Ca}^{2+}$ pathway. The $\mathrm{Wnt} / \beta$-catenin pathway is mediated by $\beta$-catenin, which accumulates in the cytoplasm in the presence of Wnt and is then transferred into the nucleus. Glycogen synthase kinase- $3 \beta$ (GSK-3 $\beta$ ) acts as a critical negative control factor in the Wnt signaling pathway. The phosphorylation of GSK-3 $\beta$ results in GSK-3 $\beta$ inactivation and blocks the ability of GSK- $3 \beta$ to phosphorylate $\beta$-catenin, thereby activating the $\mathrm{Wnt} / \beta$-catenin signaling pathway. Therefore, we hypothesized that after mechanical force is applied to chondrocytes, GSK-3 $\beta$ is phosphorylated and the Wnt/ $\beta$-catenin signaling pathway is activated, triggering chondrogenesis among chondrocytes.

In the present study, the ATDC5 cell line was selected and cultured based on its chondrogenic potential in the presence of insulin. ATDC5 cells are cells isolated from the feederindependent teratocarcinoma stem cell line AT805 (13). Compared with primary MCCs, the advantages of the ATDC5 cell line are greater stability, ease of culture and expansion, and greater humanization. The cells were subjected to mechanical stretching using a pressure-operated instrument. Subsequently, immunofluorescence, western blot analysis, quantitative PCR (qPCR), and other experimental methods were used to observe activation of the Wnt/ $\beta$-catenin signaling pathway in order to determine the effect of mechanical loading on $\beta$-catenin signaling in chondrocytes. In order to observe cartilage reconstruction after different durations of orthodontic traction, cells were subjected to mechanical loading for different amounts of time.

\section{Materials and methods}

Cell culture. ATDC5 cells (Riken Cell Bank, Tsukuba, Japan) were maintained in a log growth phase in hybrid Dulbecco's modified Eagle's medium (DMEM; Thermo Scientific Hyclone, Waltham, MA, USA) containing $10 \%$ fetal bovine serum (FBS) and $10 \mu \mathrm{g} / \mathrm{ml}$ insulin-transferrin-selenium (ITS) (both from Gibco, Grand Island, NY, USA). Cells were cultured at $37^{\circ} \mathrm{C}$ in a humidified atmosphere of $5 \% \mathrm{CO}_{2}$ in air for 21 days. Cells were initially seeded at a density of $6 \times 10^{4}$ cells/well in 6-well plates. The medium was replaced every 2 days. Alcian blue staining and qPCR analysis were used to confirm successful induction of chondrogenesis. The sequences of primers used in the present study for $\beta$-actin, collagen II, and aggrecan were as follows: $\beta$-actin sense, 5'-ATGTACGTAGCCATCCAG-3' and antisense, 5'-GAGGTAGTCTGTCAGGTC-3'; collagen II sense, 5'-CCAGAACATCACCTACCACTGTAA-3' and antisense, 5'-GCCCTCATCTCTACATCATTGGA-3'; and aggrecan sense, 5'-CCATGTGTGGGTGACAAAGACAG-3' and antisense, 5'-TCCACGTAGCAGTAGACATCATAGG-3'. These sequences were designed by Takara Bio Co., Ltd. (Kyoto, Japan).

Mechanical loading. Cells were subjected to mechanical stretching using a pressure-operated instrument (Flexercell Strain Instrument; Flexcell Corp., Hillsborough, NC, USA). Chondrocytic cells were plated in 6-well elastic-bottomed culture plates (Flex II culture plates; Flexcell Corp.), in which the bottomed surfaces of the plates were coated with collagen II. When the cells reached confluence, 4 plates were fixed on a Flexercell strain unit (Flexercell Corp.) and cyclic mechanical tension was applied. Culture plate bottoms were deformed to a known percentage of elongation, which was maximal at the edge and decreased toward the center. The strain was transferred to the cultured cells. In our experiments, the culture plate bottoms were deformed to $12 \%$ elongation in a $0.05 \mathrm{~Hz}$ cyclic ( $5 \mathrm{sec}$ on, $5 \mathrm{sec}$ off) manner. The cells were stimulated for 1,2, 4, or $8 \mathrm{~h}$, and control cells were not exposed to mechanical loading.

Protein extraction and western blot analysis. After cyclic stretch loading, cells were washed once with ice-cold phosphatebuffered saline (PBS) immediately and lysed in $500 \mu 1$ sodium dodecyl sulfate (SDS) buffer [125 mM Tris-HCL (pH 6.8), $1 \%$ $\mathrm{w} / \mathrm{v}$ SDS, $8.5 \%$ glycerol) per well. Samples were heated to $95^{\circ} \mathrm{C}$ for $5 \mathrm{~min}$. Aliquots of $10 \mu \mathrm{l}$ protein were resolved by SDS-PAGE (10\% resolving gel), transferred to nitrocellulose membranes (Bio-Rad Laboratories, Hercules, CA, USA), and blocked with blocking buffer $[10 \mathrm{mM}$ Tris- $\mathrm{HCl}(\mathrm{pH} 7.6), 150 \mathrm{mM} \mathrm{NaCl}$, $3 \% \mathrm{w} / \mathrm{v}$ BSA, $0.1 \%$ Tween-20] for $1 \mathrm{~h}$ at room temperature. After washing three times for 5 min each with Tris-buffered saline/Tween [10 mM Tris-HCl (pH 7.6), $150 \mathrm{mM} \mathrm{NaCl}, 0.1 \%$ Tween-20], cell membranes were incubated with primary antibodies overnight at $4^{\circ} \mathrm{C}$ with gentle shaking. The membranes were washed three times for $5 \mathrm{~min}$ with Tris-buffered saline/ Tween 20 and incubated with horseradish peroxidase-conjugated goat anti-rabbit secondary antibodies (1:1,000; Perbio Science, Erembodegem, Belgium) for $1 \mathrm{~h}$ at room temperature. The primary antibodies were against active $\beta$-catenin (clone 8E7, 1:1,000; Millipore, Billerica, MA, USA), phospho-GSK-3 $\beta$ (Ser9, 1:1,000; 9331S) and GSK-3ß $(1: 1,000 ; 9237$ S) (both from Cell Signaling Technology, Danvers, MA, USA). To demonstrate equal protein loading, specific $\beta$-actin antibodies $(1: 1,000 ; 4967$; Cell Signaling Technology) were used.

RNA extraction and $q P C R$. After the cells were exposed to cyclic stretch loading for $1,2,4$, or $8 \mathrm{~h}$, total RNA was extracted using TRIzol (Gibco) according to the manufacturer's instructions, and the concentration and purity were subsequently assayed by spectrophotometry. First-strand cDNA synthesis was performed using the High-Capacity cDNA Archive kit (Applied Biosystems, Foster City, CA, USA) according to the manufacturer's instructions. The sequences of primers for $\beta$-actin and collagen II were the same as those which were listed above. The sequences of primers for Wnt $3 \mathrm{a}$, disheveled homolog 1 (Dvl-1), and $\mathrm{T}$ cell-specific transcription factor (Tcf)-1 used in the present study were as follows: Wnt3a sense, 5'-CAGCTGTGAAGTGAAGAC-3' and antisense, 
5'-CTCGTAGTAGACCAGGTC-3'; Dvl-1 sense, 5'-ATC AACCAATGTCTTCAG-3' and antisense, 5'-AATGTCAA GCCAATATCC-3'; and Tcf-1 sense, 5'-CTACGAACATTTC AGCAG-3' and antisense, 5'-GTCTATATCCGCAGGAAG-3'. The sequences for all primers were designed by Takara Bio Co., Ltd. PCR was performed with the ABI Prism 7000 Sequence Detection system (Applied Biosystems). Relative quantification was carried out using the $\Delta \Delta \mathrm{Ct}$ method by taking the difference $(\Delta \mathrm{Ct})$ between the $\Delta \mathrm{Ct}$ of $\beta$-actin and the $\Delta \mathrm{Ct}$ of each transcript and computing the $\Delta \Delta \mathrm{Ct}$. Triplicate reactions were carried out in three separate experiments.

Immunofluorescence staining. After culture with or without cyclic stretch loading, cells which adhered to the surface of elastic-bottomed plates were rinsed twice with PBS, fixed with $4 \%$ paraformaldehyde for $30 \mathrm{~min}$, and rinsed twice with PBS again. The elastic bottoms of the plates were cut into 8-10 pieces each, and then cells were incubated with primary antibody (mouse anti-active $\beta$-catenin, clone 8E7, 1:100; Millipore) for $1 \mathrm{~h}$. The secondary antibody was fluorescein isothiocyanate-conjugated rabbit anti-mouse immunoglobulin G (1:200; 7074S; Cell Signaling Technology), and the stained cells were viewed through a laser scanning confocal microscope (FV1200; Olympus, Tokyo, Japan). We randomly selected six visual fields for each point and counted both the number of cells exhibiting nuclear translocation of $\beta$-catenin and the total number of cells in each field of view.

Treatment of the cells with Wnt/B-catenin signaling pathway inhibitory protein Dickkopf-related protein 1 (DKK-1). Singlecell suspensions of the chondrocytes at logarithmic growth phase were prepared, and the cells were transferred to 6-well plates. Cells were incubated with $100 \mathrm{ng} / \mathrm{ml}$ mouse recombinant Dickkopf-related protein 1 (R\&D Systems, Minneapolis, MN, USA) in DMEM containing 5\% fetal bovine serum for $24 \mathrm{~h}$ to inhibit the Wnt/ $\beta$-catenin pathway. MTT, [3-(4,5-dimethylthiazol-2-yl)-2,5-diphenyltetrazolium bromide] assay was carried out on days $1,3,5$, and 7 in cultures to evaluate the efficiency of DKK-1. Cells were plated at a density of $2 \times 10^{3}$ cells/well in 96-well plates for $4 \mathrm{~h}$ at $37^{\circ} \mathrm{C}$. The supernatant was then removed and $150 \mu 1$ dimethyl sulfoxide (DMSO) was added, followed by $10 \mathrm{~min}$ of oscillation. The optical density (OD) values for each well were measured spectrophotometrically at $490 \mathrm{~nm}$, and the tests were repeated 5 times. Control cells were not exposed to DKK-1.

To examine the effect of DKK-1 treatment on chondrocytes exposed to mechanical loading, the culture plate bottoms were deformed to $12 \%$ elongation in a $0.05 \mathrm{~Hz}$ cyclic $(5 \mathrm{sec}$ on, $5 \mathrm{sec}$ off) manner, as described above, for 1,2, 4, or $8 \mathrm{~h}$. The control group was not exposed to loading. Immunofluorescence staining was used to track $\beta$-catenin localization in chondrocytes, as also described above, after treatment with DKK-1, and $\mathrm{qPCR}$ analysis was used to detect the corresponding mRNA expression of Wnt3a, Dvl-1, Tcf-1 and collagen II, as described above. The results for cells treated with DKK-1 were compared to those for cells not treated with DKK-1.

Statistical analysis. All the experiments were repeated three times, and all statistical analyses were performed using SPSS version 16.0 software (SPSS Inc., Chicago, IL, USA). The student's t-test was employed to evaluate the significance of differences among groups, and a P-value $<0.05$ was considered to indicate a statistically significant difference.

\section{Results}

Chondrogenic differentiation and cartilage nodule formation in ATDC5 cells. ATDC5 cells can be induced to differentiate into chondrocytes in the presence of insulin (13), and a previous study has demonstrated that in the presence of $10 \mu \mathrm{g} / \mathrm{ml}$ insulin, ATDC 5 cells form cartilage nodules through a cellular condensation process that gives rise to proliferating chondrocytes (14). In the present study, the size and number of cartilage nodules within ATDC5 cultures increased each day over 3 weeks in culture after treatment with ITS. Increasing intensity of Alcian blue staining of sulfated glycosaminoglycan, which is secreted by chrondrocytes, over 3 weeks in culture confirmed chondrogenic differentiation of the ATDC5 cells (Fig. 1A).

Collagen type II and aggrecan are the main components of the extracellular matrix in cartilage. To further confirm that ATDC 5 cells differentiated into chondrocytes in the present study, we analyzed the expression of chondrogenic marker genes including collagen II and aggrecan by qPCR (Fig. 1B) upon culture of the cells in medium containing insulin. Collagen II and aggrecan expression increased from day 1 to 21, indicating that the undifferentiated ATDC5 cells had differentiated into proliferative chondrocytes.

Mechanical loading induces nuclear translocation of $\beta$-catenin in the differentiated chondrocytes. It has previously been noted that the Wnt/ $\beta$-catenin signaling pathway is mediated by $\beta$-catenin, which accumulates in the cytoplasm and translocates into the nucleus to activate Wnt signaling (15). To determine whether mechanical loading activates Wnt/ $\beta$-catenin signaling, immunofluorescence staining was used to identify dephosphorylated $\beta$-catenin in cells at $1,2,4$, and $8 \mathrm{~h}$ after stretch loading. Compared to control cells which did not receive mechanical loading, the nuclei of cells subjected to force loading for $1 \mathrm{~h}$ showed significantly increased staining for dephosphorylated $\beta$-catenin. Nuclear translocation of $\beta$-catenin occurred after $1 \mathrm{~h}$, and the amount of nuclear staining in cells subjected to stretch loading for $8 \mathrm{~h}$ was comparable to that in the control group (Fig. 2B). These results indicate that translocation of $\beta$-catenin is a rapid process.

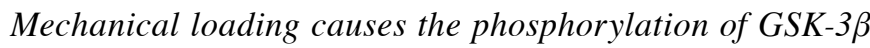
and the accumulation of $\beta$-catenin. GSK-3 $\beta$ is a key component of the Wnt/ $\beta$-catenin signaling pathway, and its activity is inhibited by phosphorylation at serine (16). GSK-3 $\beta$ is normally inhibited in the Wnt pathway, where its primary target is $\beta$-catenin. Phosphorylation of GSK-3 $\beta$ inhibits its ability to phosphorylate $\beta$-catenin, which marks $\beta$-catenin for degradation (17). Therefore, we hypothesized that upon mechanical loading, GSK-3 $\beta$ in the chondrocytes would be phosphorylated, thereby activating the $\mathrm{Wnt} / \beta$-catenin signaling pathway. Western blot analysis showed that the level of p-GSK-3 $\beta$ increased with the application of stretch loading, and this increase was accompanied by an increase in the level of dephosphorylated $\beta$-catenin at 1 and $2 \mathrm{~h}$ after application of mechanical loading (Fig. 3). 

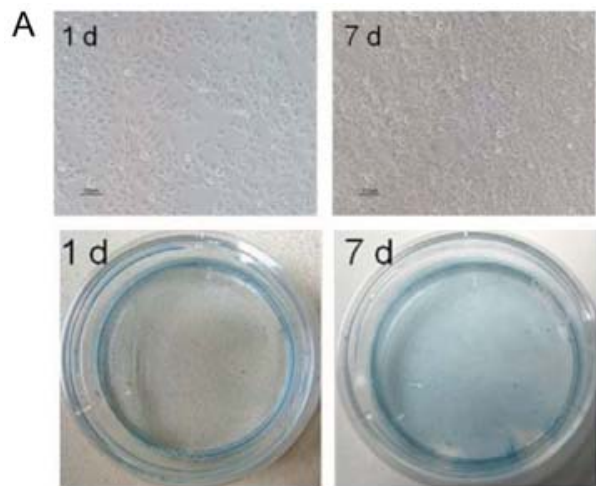

$7 \mathrm{~d}$

B

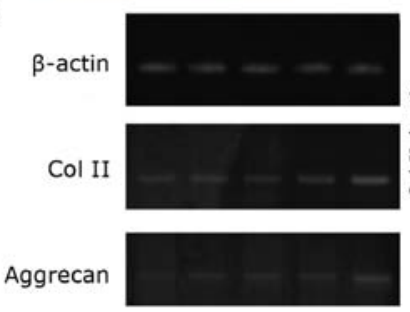

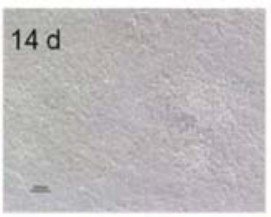
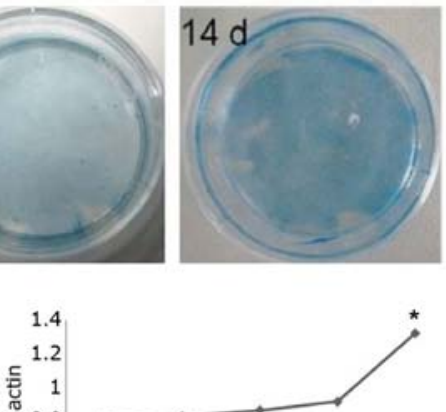

$\stackrel{0}{*} 0.8$

0.2

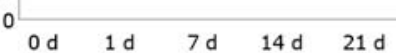

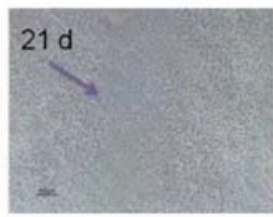
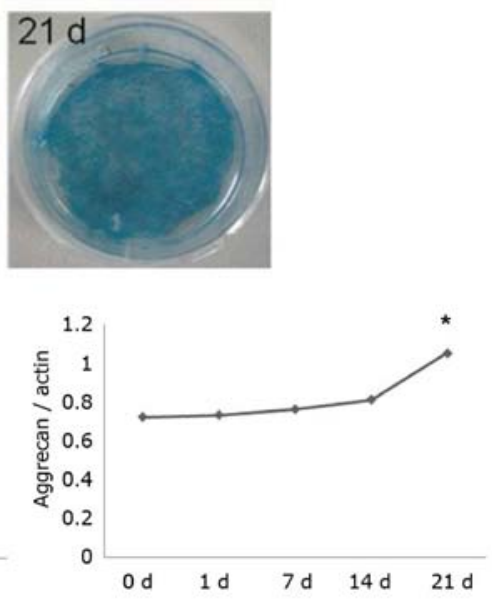

Figure 1. (A) The images in the first row are phase-contrast micrographs of ATDC5 cells over 3 weeks in culture with insulin treatment. The arrow indicates a cartilage nodule. Scale bar, $100 \mu \mathrm{m}$. The second row contains images of ATDC5 cell differentiation in culture. Alcian blue staining of sulfated glycosaminoglycan accumulated over 3 weeks of ATDC5 cell culture with insulin. (B) ATDC5 cells were cultured in DMEM containing insulin for 21 days. The mRNA expression levels of chondrogenic markers collagen II and aggrecan in ATDC5 cells were measured by qPCR and normalized to the expression of $\beta$-actin. The experiment was repeated three times. Data represent the means $\pm \mathrm{SD}$. The results showed that there was a significant increase in collagen II and aggrecan gene expression. (Scale bar, $100 \mu \mathrm{m}$ ). ${ }^{*} \mathrm{P}<0.01$. D, days.
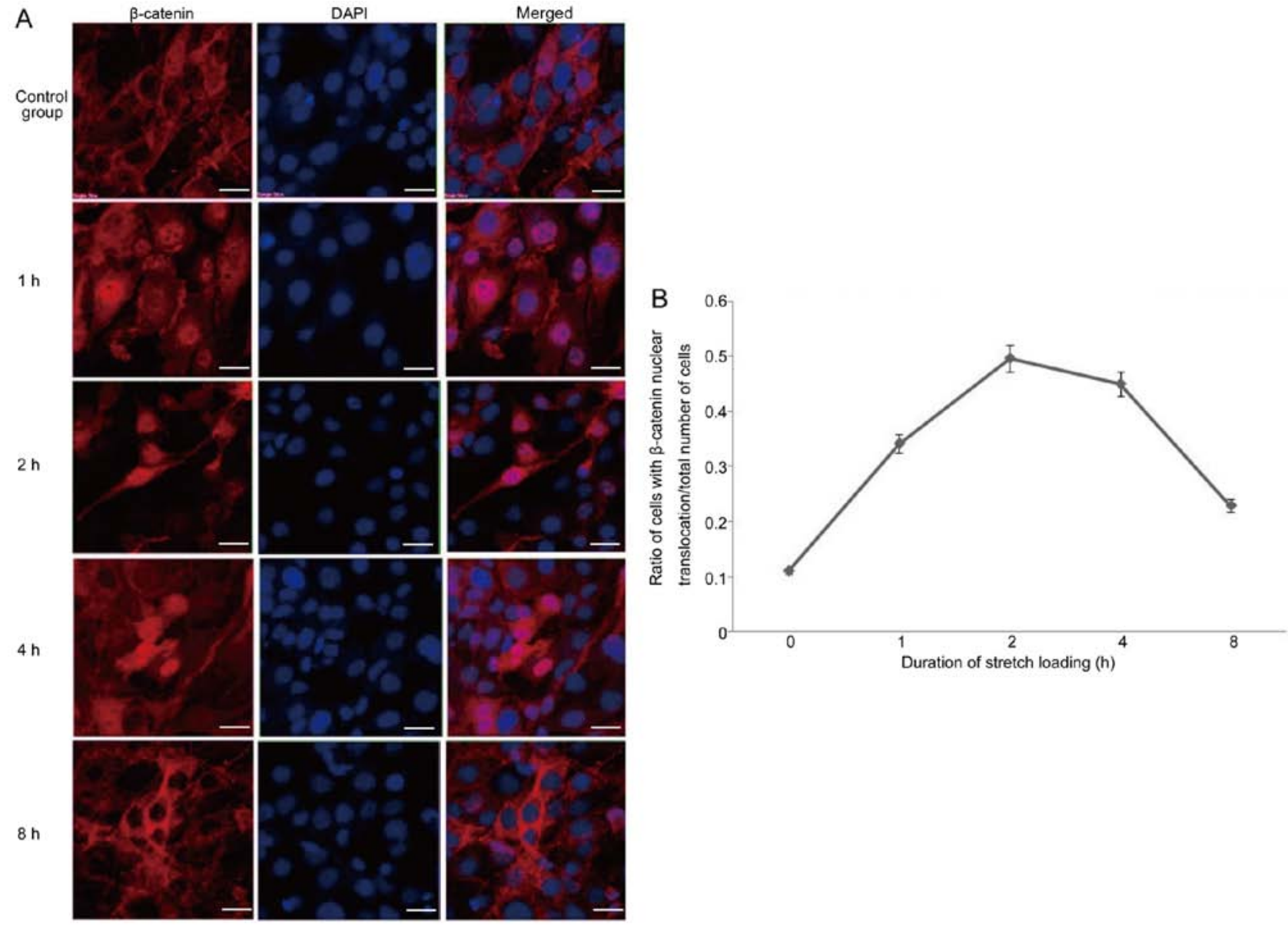

Figure 2. (A) Mechanical loading-induced nuclear translocation of $\beta$-catenin. Cells were exposed to $12 \%$ cycle mechanical stretching for $1,2,4$, and $8 \mathrm{~h}$. Immunofluorescence staining was performed to observe the location of dephosphorylated $\beta$-catenin, and DAPI was used to stain the nuclei (blue). (B) Ratio of the number of cells showing $\beta$-catenin nuclear translocation to the total number of cells in six visual fields of immunofluorescence-stained cells. These stained cells were selected randomly at each time point. Data represent the means \pm SD. (Scale bar, $10 \mu \mathrm{m}$ ). 


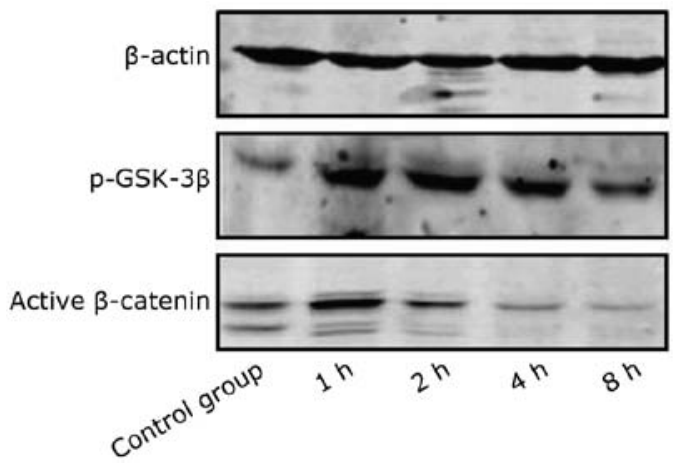

Figure 3. Western blot analysis shows increased levels of p-glycogen synthase

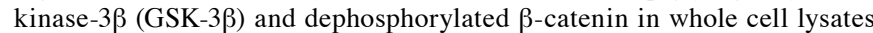
from $6 \times 10^{4}$ cells/well after 1 and $2 \mathrm{~h}$ of mechanical loading. $\beta$-actin was used as a control to confirm equal protein loading.

Wnt3a, Dvl-1, Tcf-1, and collagen II mRNA expression in chondrocytes subjected to mechanical loading. To investigate the role of the $\mathrm{Wnt} / \beta$-catenin pathway in chondrogenic differentiation, we simultaneously examined the expression of key proteins in the Wnt/ $\beta$-catenin pathway (Wnt3a, Dvl-1, and Tcf-1) as well as collagen II, as a marker of differentiation. After $2 \mathrm{~h}$ of mechanical loading, the expression of Wnt3a mRNA was significantly higher than that in the control group, whereas after $4 \mathrm{~h}$ of mechanical loading, Wnt3a mRNA expression was almost equal to that in the $8 \mathrm{~h}$ group. Wnt3a expression increased initially and then decreased. Dvl mRNA expression showed the same trend. However, the expression of Tcf-1 mRNA increased and remained at a high level almost equivalent to that after $1 \mathrm{~h}$ over the entire $8 \mathrm{~h}$ of mechanical loading. Collagen II expression followed a similar trend as Tcf-1, increasing and then remaining at a high level, but decreased at $8 \mathrm{~h}$ (Fig. 4).
Effects of the inhibitor DKK-1 on Wnt/ $\beta$-catenin pathway activation in chondrocytes subjected to mechanical loading. Wnt signaling is tightly regulated by members of several families of secreted antagonists (18). DKK-1 is a natural regulator of the Wnt/ $\beta$-catenin signaling pathway that has the ability to specifically block the pathway $(19,20)$. In the present study, we applied DKK-1 to differentiated ATDC5 cells to further examine the role of the Wnt/ $\beta$-catenin signaling pathway in these cells when subjected to mechanical loading. According to the results of the MTT assay, chondrocytes showed restricted growth after treatment with DKK-1 (Fig. 5). Immunofluorescence staining demonstrated an obvious decrease in the amount of dephosphorylated $\beta$-catenin in the nuclei of cells subjected to stretch loading and exposed to DKK-1 compared to cells subjected to stretch loading only (Fig. 6). In accordance with these results, western blot analysis also demonstrated that exposure to DKK-1 generally decreased the level of dephosphorylated $\beta$-catenin in whole cell lysate. The levels of $\mathrm{p}-\mathrm{GSK}-3 \beta$ were increased slightly after mechanical loading and also DKK-1 treatment (Figure 7), compared with levels in cells not exposed to DKK-1 (Figure 5). These changes in p-GSK-3 $\beta$ levels correlated with those in levels of dephosphorylated $\beta$-catenin at $1 \mathrm{~h}$ (Fig. 7). The expression of Wnt3a, Dvl-1, Tcf-1, and collagen II mRNA were also suppressed by DKK-1 (Fig. 8).

\section{Discussion}

Mechanical loading is an important regulator of chondrocyte function, but the mechanisms by which mechanical stimulation influences intracellular signaling are still not fully understood. In orthodontics, more attention is paid to the TMJ cartilage, and therapeutic corrections are applied to stimulate cell and tissue remodeling by manipulation using mechanical forces (21). In the present study, we found that the $\mathrm{Wnt} / \beta$-catenin signaling
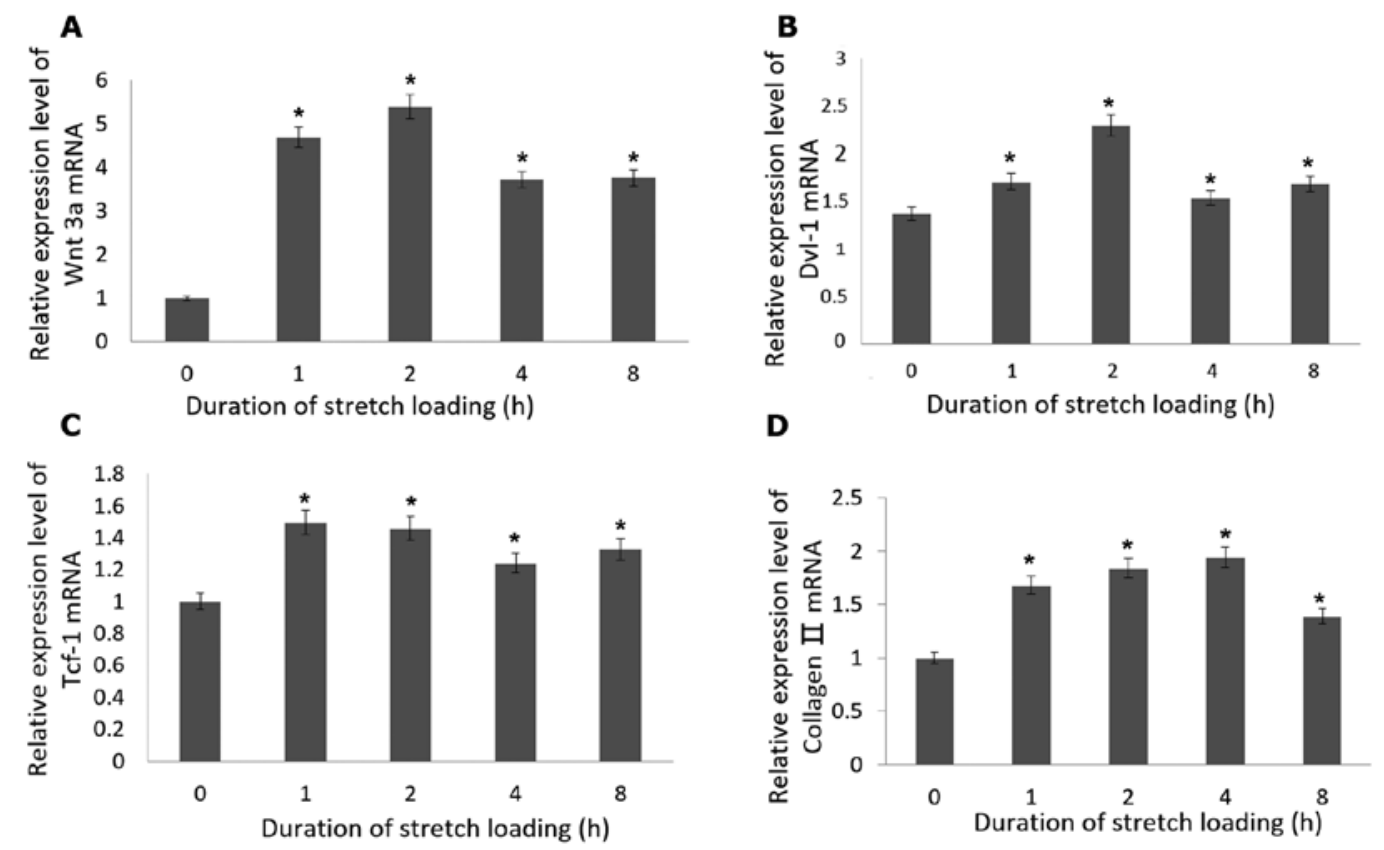

Figure 4. Relative expression of (A) Wnt3a, (B) disheveled homolog 1 (Dvl-1), (C) T cell-specific transcription factor-1 (Tcf-1), and (D) collagen II mRNA over an 8-h period of mechanical loading. After stretch loading at 1, 2, 4, $8 \mathrm{~h}$, there was a significant increase in the gene expression for Wnt3a, Dvl-1, Tcf-1 and collagen II compared with that at the initial time. ${ }^{*} \mathrm{P}<0.05$. 


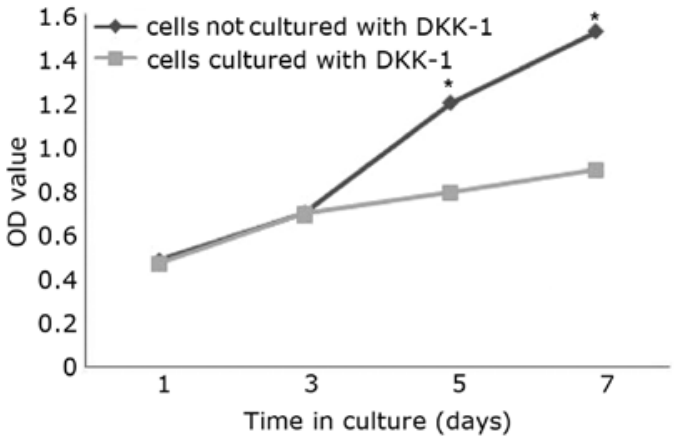

Figure 5. MTT assay of chondrocytes exposed and not exposed to Dickkopf-related protein 1 (DKK-1), demonstrating that DKK-1 effectively restricted cell growth after the third day. ${ }^{*} \mathrm{P}<0.01$.

pathway is activated in chondrocytes upon application of mechanical loading.

GSK-3 $\beta$ is an important negative control factor in the Wnt signaling pathway, and phosphorylation of GSK-3 $\beta$ inactivates GSK-3 $\beta$ and activates the Wnt $/ \beta$-catenin signaling pathway (18). Thus, we hypothesized that after mechanical force acts upon chondrocytes, GSK- $3 \beta$ is phosphorylated to activate the Wnt/ $\beta$-catenin signaling pathway and trigger chondrogenesis. In our study, chondrocytes were exposed to a $12 \%$ cycle mechanical stretching for $1,2,4$, and $8 \mathrm{~h}$, which simulated orthodontic traction. Via immunofluorescence staining, we observed that $\beta$-catenin translocated from the cytoplasm to the nucleus after $1 \mathrm{~h}$ of mechanical loading, indicating activation of the Wnt/ $\beta$-catenin signaling pathway. However, by $8 \mathrm{~h}$ of mechanical loading, the proportion of cells demonstrating nuclear translocation of $\beta$-catenin had declined, indicating rapid changes in the activity of this protein. More detailed analyses of these protein events are necessary to improve our understanding of cartilage physiology and pathology.

GSK-3 $\beta$ constitutively phosphorylates the $\mathrm{N}$-terminus of $\beta$-catenin cyclin-dependent kinase at Ser, which marks $\beta$-catenin for degradation. When GSK-3 $\beta$ is phosphorylated at Ser, $\beta$-catenin accumulates in the cytoplasm before translocating to the nucleus to activate the Wnt/ $\beta$-catenin signaling pathway (18). According to western blot analysis, active dephosphorylated $\beta$-catenin and p-GSK-3 $\beta$ protein expression increased in chondrocytes in response to mechanical strain, indicating activation of the Wnt/ $\beta$-catenin signaling pathway as well as simultaneous inactivation of GSK- $3 \beta$ by mechanical loading.
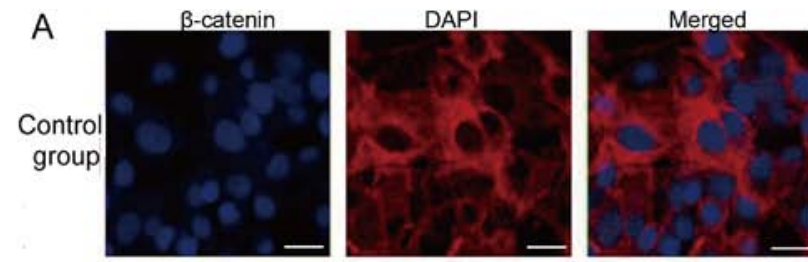

$1 \mathrm{~h}$
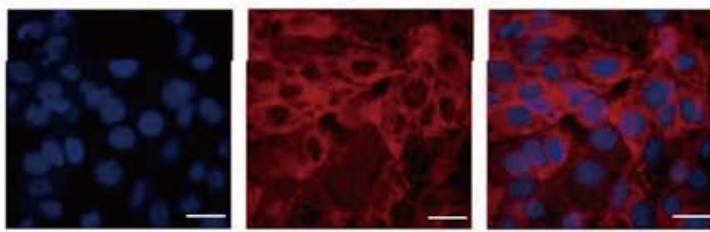

$2 \mathrm{~h}$
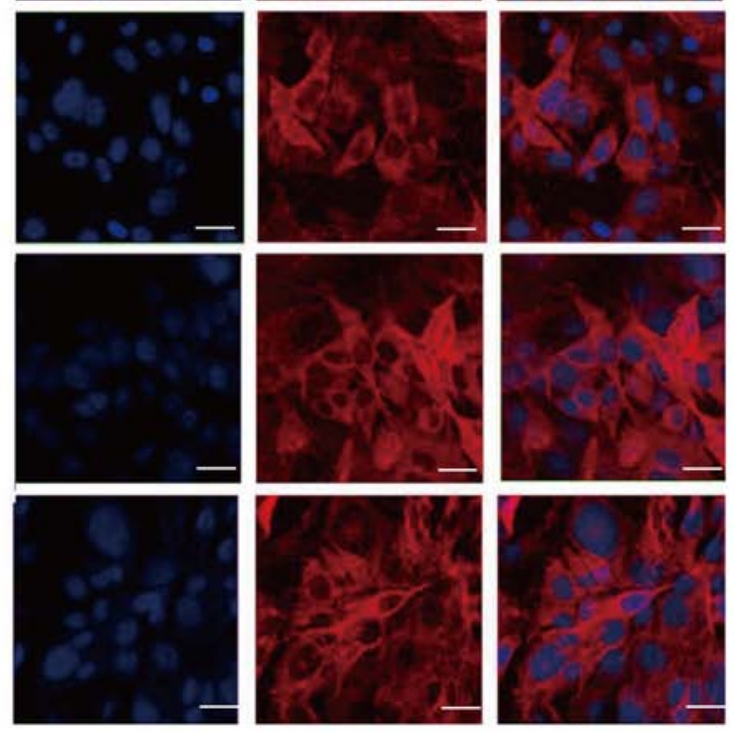

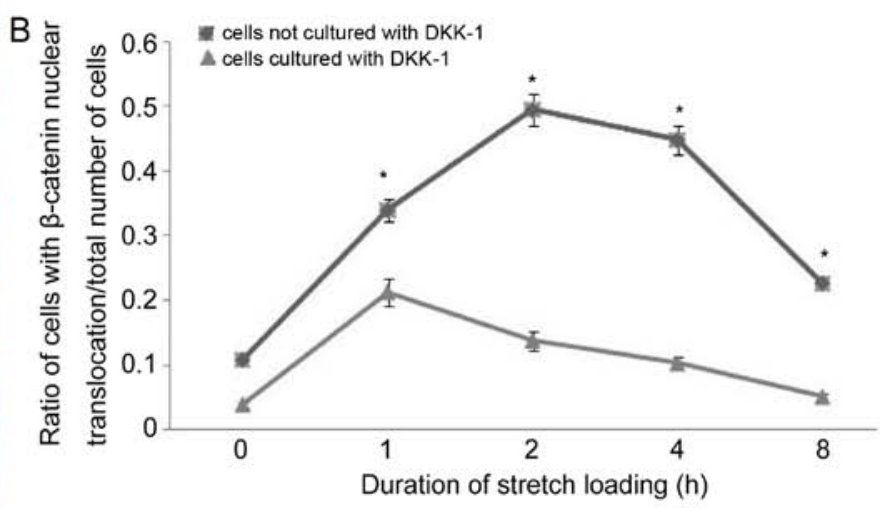

Figure 6. (A) Immunofluorescence staining of dephosphorylated $\beta$-catenin in chondrocytes exposed to stretch loading and DKK-1. After application of DKK-1, the proportion of cells showing nuclear translocation of $\beta$-catenin was obviously decreased, indicating suppression of the Wnt/ $\beta$-catenin signaling pathway. DAPI was used to stain the nuclei (blue). (B) Ratio of the number of cells showing $\beta$-catenin nuclear translocation to the total number of cells in six randomly selected visual fields of cells immunofluorescently stained for $\beta$-catenin with or without exposure to DKK-1. (Scale bar, $10 \mu \mathrm{m}$ ). There was a marked decrease in the amount of dephosphorylated $\beta$-catenin in the nuclei of cells subjected to stretch loading and exposed to DKK- 1 compared to the cells subjected to stretch loading only. ${ }^{*}<0.01$. 


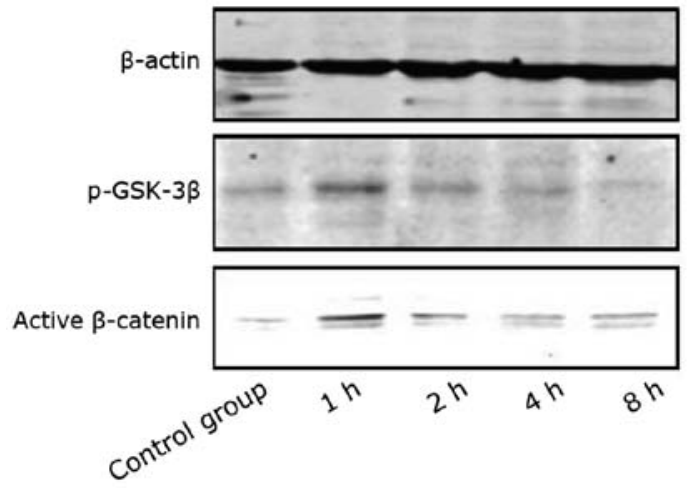

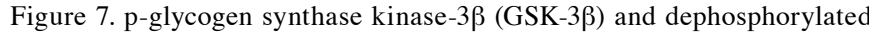
$\beta$-catenin protein expression as detected by western blot analysis. p-GSK-3 $\beta$ and dephosphorylated $\beta$-catenin levels in whole cell lysates of cells exposed to DKK-1 were slightly elevated after $1 \mathrm{~h}$ of mechanical loading but much lower than levels in cells not treated with DKK-1 (Figure 5). $\beta$-actin was used as a control to confirm equal protein loading.

The Wnt family proteins play important roles in patterning and cell fate determination; Wnt 4 and Wnt9a are expressed in the developing joints (22). Wnt3a promotes chondrogenic differentiation but delays hypertrophy $(11,23)$. The Wnt family binds to a complex consisting of the Frizzled family of transmembrane receptors and the lipoprotein receptor-related protein (LRP) family of co-receptors (24). Wnt3a is thought to be a 'canonical' Wnt protein, signaling through a $\beta$-catenin-dependent signaling pathway. In the absence of the Wnt signal, Tcf acts as a repressor of Wnt target genes by forming a complex with Groucho (25). When $\beta$-catenin translocates to the nucleus, $\beta$-catenin interacts with transcription factors such as Tcf to affect the transcription of target genes (26). It has previously been noted that the physiological dynamic load induces the synthesis of collagen II, and that the absence of this load leads to a reduction in collagen II synthesis (27). In the present study, qPCR analysis was used to evaluate the mRNA expression of Wnt3a, Dvl-1, Tcf-1, and collagen II in chondrocytes exposed to mechanical strain. The increased expression of these key proteins in the Wnt/ $\beta$-catenin signaling pathway suggests that mechanical loading induced activation of $\beta$-catenin in chondrocytes.

DKK-1 has been noted to specifically affect the canonical Wnt signaling pathway by binding and modulating Wnt coreceptors of LRP5/6 (28). Therefore, we used DKK-1 to inhibit mechanical loading-induced dephosphorylation of $\beta$-catenin, and the results showed that treatment with DKK-1 generally decreased the levels of dephosphorylated $\beta$-catenin and p-GSK-3 $\beta$ in whole cell lysates. The expression levels of Wnt3a, Dvl-1, Tcf-1, and collagen II mRNA were also suppressed with addition of DKK-1 but were still increased and then declined by exposure to mechanical loading. Thus, the mechanical loadinginduced activation of $\beta$-catenin in chondrocytes is not only mediated by Wnt/ $\beta$-catenin receptors. This finding is in agreement with those of previous studies on periodontal ligament cells and osteoblasts $(17,29)$, but the mechanism underlying phosphorylation of GSK-3 $\beta$ requires further research.

It is well known that physiological dynamic loads induce the synthesis of collagen II, whereas a static load has the opposite effect $(10,30)$. The increase in collagen II expression upon the application of mechanical strain in the present study further confirms the close association between mechanical stain and collagen II production by chondrocytes. In addition,
A
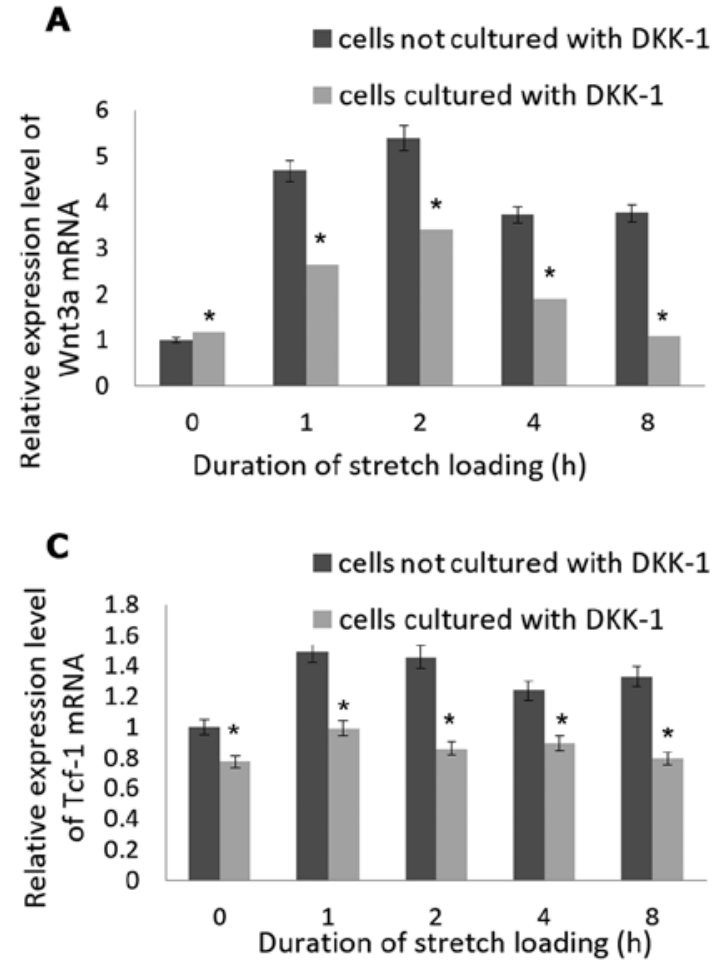

B
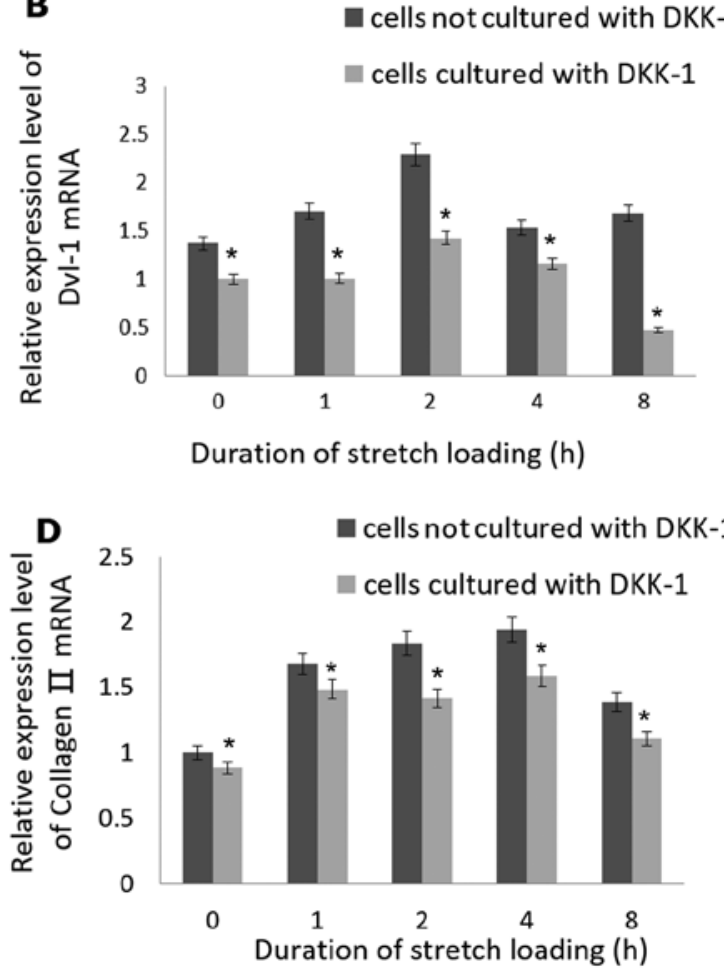

Figure 8. Expression of (A) Wnt3a, (B) disheveled homolog 1 (Dvl-1), (C) T cell-specific transcription factor-1 (Tcf-1), and (D) collagen II mRNA after application of inhibitor DKK-1 to chondrocytes subjected to stretch loading. The mRNA expression of Wnt3a, Dvl-1, Tcf-1, and collagen II was also suppressed by DKK-1. ${ }^{*} \mathrm{P}<0.05$ 
the simultaneous increases in GSK-3 $\beta$ phosphorylation and Wnt/ $\beta$-catenin signaling pathway activation indicate that the Wnt/ $\beta$-catenin signaling pathway plays an important role in the process of collagen II upregulation induced by mechanical loading. Therefore, we suggest that the Wnt/ $\beta$-catenin signaling pathway represents a new therapeutic target for inducing cartilage formation in the field of regenerative medicine. In the field of oral medicine, specifically, these findings should be applied to the development of novel strategies for TMJ reconstruction and regeneration, which are necessary for the treatment of TMJ disorders and to guide orthodontic treatments.

In conclusion, the present research first demonstrated that mechanical loading induced the translocation of active $\beta$-catenin from the cytoplasm to the nucleus in chondrocytes. Thus, we suggest that $\beta$-catenin plays an important role in the general response of chondrocytes to mechanical strain. Overall, our results indicate that the Wnt/ $\beta$-catenin signaling pathway is functional and activated by mechanical strain in chondrocytes.

\section{References}

1. Kinzinger G, Kober $\mathrm{C}$ and Diedrich P: Topography and morphology of the mandibular condyle during fixed functional orthopedic treatment - a magnetic resonance imaging study.J Orofac Orthop 68: 124-147, 2007.

2. Bewyer DC: Biomechanical and physiologic processes leading to internal derangement with adhesion. J Craniomandib Disord 3: 44-49, 1989.

3. Meikle MC: Remodeling the dentofacial skeleton: the biological basis for orthodontics and dentofacial orthopedics. J Dent Res 86: $12-24,2007$.

4. Habib H, Hatta T, Udagawa J, Zhang L, Yoshimura Y and Otani H: Fetal jaw movement affects condylar cartilage development. J Dent Res 84: 474-479, 2005.

5. Madhavan S, Anghelina M, Rath-Deschner B, Wypasek E, John A, Deschner J, Piesco N and Agarwal S: Biomechanical signals exert sustained attenuation of proinflammatory gene induction in articular chondrocytes. Osteoarthritis Cartilage 14: 1023-1032, 2006.

6. Ker RF, Wang XT and Pike AV: Fatigue quality of mammalian tendons. J Exp Biol 203: 1317-1327, 2000.

7. Kuboki T, Shinoda M, Orsini MG and Yamashita A: Viscoelastic properties of the pig temporomandibular joint articular soft tissues of the condyle and disc. J Dent Res 76: 1760-1769, 1997.

8. Kronenberg HM: Developmental regulation of the growth plate. Nature 423: 332-336, 2003.

9. Maruyama T, Mirando AJ, Deng CX and Hsu W: The balance of WNT and FGF signaling influences mesenchymal stem cell fate during skeletal development. Sci Signal 3: ra40, 2010.

10. Larsson T, Aspden RM and Heinegård D: Effects of mechanical load on cartilage matrix biosynthesis in vitro. Matrix 11: 388-394, 1991.

11. Church V, Nohno T, Linker C, Marcelle C and Francis-West P: Wnt regulation of chondrocyte differentiation. J Cell Sci 115: 4809-4818, 2002.

12. Chimal-Monroy J, Montero JA, Gañan Y, Macias D, Garcia-Porrero JA and Hurle JM: Comparative analysis of the expression and regulation of Wnt5a, Fz4, and Frzb1 during digit formation and in micromass cultures. Dev Dyn 224: 314-320, 2002.
13. Atsumi T, Miwa Y, Kimata K and Ikawa Y: A chondrogenic cell line derived from a differentiating culture of AT805 teratocarcinoma cells. Cell Differ Dev 30: 109-116, 1990.

14. Shukunami C, Shigeno C, Atsumi T, Ishizeki K, Suzuki F and Hiraki Y: Chondrogenic differentiation of clonal mouse embryonic cell line ATDC5 in vitro: differentiation-dependent gene expression of parathyroid hormone $(\mathrm{PTH}) / \mathrm{PTH}$-related peptide receptor. J Cell Biol 133: 457-468, 1996.

15. Saito-Diaz K, Chen TW, Wang X, Thorne CA, Wallace HA, Page-McCaw A and Lee E: The way Wnt works: components and mechanism. Growth Factors 31: 1-31, 2013.

16. Gould TD and Manji HK: Glycogen synthase kinase-3: a putative molecular target for lithium mimetic drugs. Neuropsychopharmacology 30: 1223-1237, 2005.

17. Case N, Ma M, Sen B, Xie Z, Gross TS and Rubin J: Beta-catenin levels influence rapid mechanical responses in osteoblasts. J Biol Chem 283: 29196-29205, 2008.

18. Liu F, Kohlmeier S and Wang CY: Wnt signaling and skeletal development. Cell Signal 20: 999-1009, 2008.

19. Aguilera O, Fraga MF, Ballestar E, Paz MF, Herranz M, Espada J, García JM, Muñoz A, Esteller M and González-Sancho JM: Epigenetic inactivation of the Wnt antagonist DICKKOPF-1 (DKK-1) gene in human colorectal cancer. Oncogene 25: 4116-4121, 2006.

20. González-Sancho JM, Aguilera O, García JM, Pendás-Franco N, Peña C, Cal S, García de Herreros A, Bonilla F and Muñoz A: The Wnt antagonist DICKKOPF-1 gene is a downstream target of beta-catenin/TCF and is downregulated in human colon cancer. Oncogene 24: 1098-1103, 2005.

21. Alanen P: Occlusion and temporomandibular disorders (TMD): still unsolved question? J Dent Res 81: 518-519, 2002.

22. Hartmann C and Tabin CJ: Dual roles of Wnt signaling during chondrogenesis in the chicken limb. Development 127: 3141-3159, 2000.

23. Rudnicki JA and Brown AM: Inhibition of chondrogenesis by Wnt gene expression in vivo and in vitro. Dev Biol 185: 104-118, 1997.

24. Yano F, Kugimiya F, Ohba S, Ikeda T, Chikuda H, Ogasawara T, Ogata N, Takato T, Nakamura K, Kawaguchi H and Chung UI: The canonical Wnt signaling pathway promotes chondrocyte differentiation in a Sox9-dependent manner. Biochem Biophys Res Commun 333: 1300-1308, 2005.

25. Brannon M, Gomperts M, Sumoy L, Moon RT and Kimelman D: A beta-catenin/XTcf-3 complex binds to the siamois promoter to regulate dorsal axis specification in Xenopus. Genes Dev 11: 2359-2370, 1997.

26. van de Wetering M, Cavallo R, Dooijes D, van Beest M, van Es J, Loureiro J, Ypma A, Hursh D, Jones T, Bejsovec A, et al: Armadillo coactivates transcription driven by the product of the Drosophila segment polarity gene dTCF. Cell 88: 789-799, 1997.

27. Ratcliffe A, Billingham ME, Saed-Nejad F, Muir H and Hardingham TE: Increased release of matrix components from articular cartilage in experimental canine osteoarthritis. J Orthop Res 10: 350-358, 1992

28. Krupnik VE, Sharp JD, Jiang C, Robison K, Chickering TW, Amaravadi L, Brown DE, Guyot D, Mays G, Leiby K, et al: Functional and structural diversity of the human Dickkopf gene family. Gene 238: 301-313, 1999.

29. Premaraj S, Souza I and Premaraj T: Mechanical loading activates $\beta$-catenin signaling in periodontal ligament cells. Angle Orthod 81: 592-599, 2011.

30. Rizkalla G, Reiner A, Bogoch E and Poole AR: Studies of the articular cartilage proteoglycan aggrecan in health and osteoarthritis. Evidence for molecular heterogeneity and extensive molecular changes in disease. J Clin Invest 90: 2268-2277, 1992. 